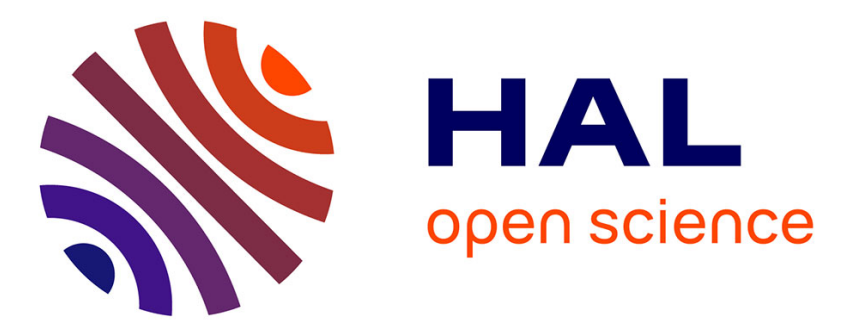

\title{
Localization of a small change in a multiple scattering environment without modeling of the actual medium
}

\author{
Sandrine Rakotonarivo, S.C. Walker, W. A. Kuperman, Philippe Roux
}

\section{To cite this version:}

Sandrine Rakotonarivo, S.C. Walker, W. A. Kuperman, Philippe Roux. Localization of a small change in a multiple scattering environment without modeling of the actual medium. Journal of the Acoustical Society of America, 2011, 130, pp.3566. 10.1121/1.3652859 . hal-01237329

\section{HAL Id: hal-01237329 \\ https://hal.science/hal-01237329}

Submitted on 29 Mar 2016

HAL is a multi-disciplinary open access archive for the deposit and dissemination of scientific research documents, whether they are published or not. The documents may come from teaching and research institutions in France or abroad, or from public or private research centers.
L'archive ouverte pluridisciplinaire HAL, est destinée au dépôt et à la diffusion de documents scientifiques de niveau recherche, publiés ou non, émanant des établissements d'enseignement et de recherche français ou étrangers, des laboratoires publics ou privés. 


\title{
Localization of a small change in a multiple scattering environment without modeling of the actual medium
}

\author{
S. T. Rakotonarivo, ${ }^{\text {a) }}$ S. C. Walker, and W. A. Kuperman \\ Scripps Institution of Oceanography, University of California San Diego, 9500 Gilman Drive, La Jolla, \\ California 92093-0923 \\ P. Roux \\ Institut des Sciences de la Terre, Université Joseph Fourier, Centre National de la Recherche Scientifique, \\ Unité Mixte de Recherche 5275, 1381 Rue de la Piscine, Saint-Martin d'Hères, France
}

(Received 5 January 2011; revised 6 September 2011; accepted 16 September 2011)

\begin{abstract}
A method to actively localize a small perturbation in a multiple scattering medium using a collection of remote acoustic sensors is presented. The approach requires only minimal modeling and no knowledge of the scatterer distribution and properties of the scattering medium and the perturbation. The medium is ensonified before and after a perturbation is introduced. The coherent difference between the measured signals then reveals all field components that have interacted with the perturbation. A simple single scatter filter (that ignores the presence of the medium scatterers) is matched to the earliest change of the coherent difference to localize the perturbation. Using a multi-source/receiver laboratory setup in air, the technique has been successfully tested with experimental data at frequencies varying from 30 to $60 \mathrm{kHz}$ (wavelength ranging from 0.5 to $1 \mathrm{~cm}$ ) for $\mathrm{cm}$-scale scatterers in a scattering medium with a size two to five times bigger than its transport mean free path. (C) 2011 Acoustical Society of America. [DOI: 10.1121/1.3652859]
\end{abstract}

PACS number(s): 43.20.Gp, 43.20.Fn [PEI]

Pages: $3566-3573$

\section{INTRODUCTION}

The single scattering approximation is not sufficient for imaging a target or a change in a multipath environment where the transport mean free path is smaller than the size of the scattering medium. To recover a change or a target location from remote sensing data, one can do an inversion using a full scattering model that describes all multiple interactions with the medium. ${ }^{1}$ However, in areas of nondestructive testing, ${ }^{2,3}$ biomedical imaging, ${ }^{4,5}$ underwater acoustics, ${ }^{6,7}$ and seismology,${ }^{8,9}$ or for a specific application as target tracking in a forest, ${ }^{10}$ the multipath environment is usually not entirely known; thereby making it difficult to model the full multi-scattered field.

Another approach is to perform an inversion using a partial scattering model derived from the available information and extract that part of the data that fits this model to locate the perturbation. Noting the phase coherent nature of wave propagation through multiple scattering media, some information is still present in the scattered field that may be used to detect, locate, or characterize a target. ${ }^{3}$ For example, the scattering cross section of a moving target in a cavity can be determined from the decay of the coherent acoustic intensity over many realizations of the scattering medium. ${ }^{11,12}$ Coda wave interferometry methods ${ }^{8,13,14}$ detect a coherent change by implementing cross-correlation between a perturbed and unperturbed field. This approach is used in conjunction with a diffusive model to locate a perturbation embodied in a multiple scattering environment. ${ }^{2}$ Finally, a coherent change can also

\footnotetext{
a) Author to whom correspondence should be addressed. Electronic mail: srakotonarivo@ucsd.edu
}

be detected by taking the difference between a perturbed and unperturbed field. ${ }^{1,10}$ Based on the analysis of this coherent difference, Liu et al. ${ }^{15}$ presented a method to locate a perturbation that requires prior measurement and estimation of the scattering medium Green's functions. Alternatively, Aubry and Derode ${ }^{4,5}$ described a method to detect and locate a strong target without modeling the multiple scattering medium, but their technique is not sensitive to a weak perturbation.

This paper presents a technique to locate a local change in the medium, which represents the target. The change may be as weak as the individual scatterers from the scattering medium, and the method does not require any prior knowledge or estimation of the multiple scattering medium properties. The approach is based on the analysis of the coherent change defined as the difference between a perturbed and unperturbed field. This subtraction operation coherently suppresses most of scattering contribution from the medium and brings out scattering interactions with the perturbation. The data for this latter process are obtained by either introducing/removing a new scatterer or by the change in position of one scatterer in the scattering medium. As a result, the earliest change occurring in the time domain subtracted field corresponds to the single scattering interaction with the disturbance. Starting from this consideration, locating the perturbation is achieved by inverting the data using a single scattering model from this earliest change. In particular, a single scattering model is constructed from a free field model between the source and the perturbation and between the perturbation and the receiver. The method is tested on a multi-source/multi-receiver laboratory set-up in air. As shown in Fig. 1, many sources and receivers surround the multiple scattering medium in which the local perturbation is occurring. 
a)

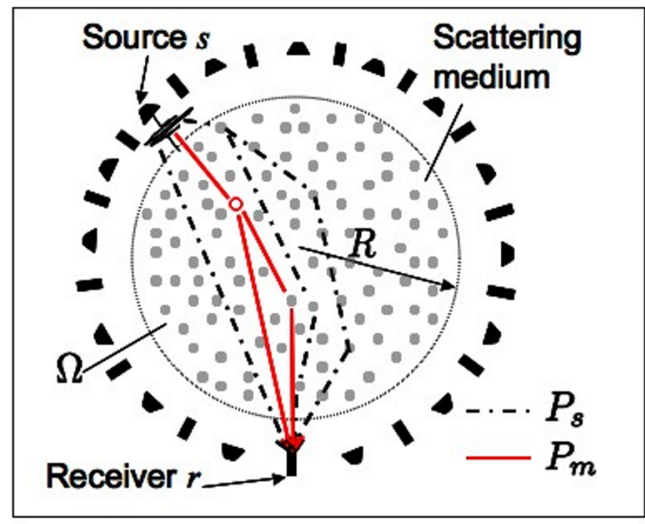

b)

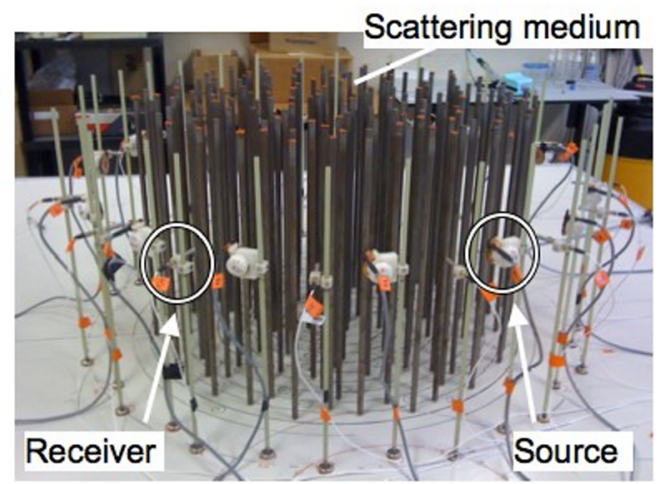

FIG. 1. (Color online) (a) Schematic of the experimental setup from above. Sources and receivers surround the propagation medium. The plain disks represent rods randomly distributed inside a disk of radius $R$ and area $\Omega$. The solid line circle represents the local perturbation. Each source $s$ transmits a broadband pulse. The measured field at receiver $r$ contains contribution from the direct field and the (single or multi-) scattered field, which has only interacted with fixed rods from the scattering medium (dotted dashed line) and from the (single or multi-) scattered field which has interacted with the perturbation (solid lines). (b) Picture of the multi-source/multi-receiver laboratory setup in air.

The structure of the paper is as follows. Section II presents a theoretical basis for the method to detect and locate a perturbation caused by either introducing or removing a scatterer or by moving one scatterer in the scattering medium. We show that the first change of the subtracted field is related to the single scattered wave. A signal processing method to locate the target is also described. Section III presents the experimental setup and results. Next, results are compared and discussed in Sec. IV and conclusions are presented in Sec. V.

\section{LOCATING A COHERENT CHANGE IN A SCATTERING MEDIUM}

\section{A. Coherent change from the subtracted field}

A point source at the location $\mathbf{r}_{s}$ transmits a cylindrical wave to the 2-D scattering medium composed of $N$ randomly distributed scatterers. The source, receiver, and scatterers are assumed to be in the far-field of each other. The relative size of the scatterers is of the same order as the wavelength with $k a \geq 1$, where $k$ is the wave number and $a$ is the scatterer characteristic length. To locate a small perturbation occurring in such a scattering medium, the pressure field from the scattering medium at position $\mathbf{r}$ is measured twice, before

and after the perturbation has occurred. The fields measured before and after the perturbation are called the unperturbed and perturbed fields, respectively. The subtracted field is then calculated as

$$
\Delta P\left(\mathbf{r}, \mathbf{r}_{s}\right)=P^{(1)}\left(\mathbf{r}, \mathbf{r}_{s}\right)-P^{(2)}\left(\mathbf{r}, \mathbf{r}_{s}\right),
$$

where $P^{(i)}\left(\mathbf{r}, \mathbf{r}_{s}\right)$ represents the field at a distant receiver $\mathbf{r}$ from a source at $\mathbf{r}_{s} ; i=1$ refers to the unperturbed field and $i=2$ refers to the perturbed field.

Following the notation in Fig. 1(a), the pressure field is divided into two terms:

$$
P^{(i)}\left(\mathbf{r}, \mathbf{r}_{s}\right)=P_{s}\left(\mathbf{r}, \mathbf{r}_{s}\right)+P_{m}^{(i)}\left(\mathbf{r}, \mathbf{r}_{s}\right),
$$

where $P_{m}^{(i)}$ represents the scattered field that has interacted with the perturbation and $P_{s}$ represents the scattered field that has not interacted with the perturbation and remains constant over the unperturbed and perturbed configurations. Subtracting fields $P^{(1)}$ and $P^{(2)}$ suppresses the constant wavefield contribution, which has not interacted with the perturbation, so that

$$
\Delta P\left(\mathbf{r}, \mathbf{r}_{s}\right)=P_{m}^{(1)}\left(\mathbf{r}, \mathbf{r}_{s}\right)-P_{m}^{(2)}\left(\mathbf{r}, \mathbf{r}_{s}\right)
$$

The scattered field $P_{m}^{(i)}$ is the only remaining component after subtraction and is made of single and multiple scattering events, which have interacted with the perturbation. Using recursive relations from multiple scattering theory, ${ }^{16-18}$ the derivation of the subtracted field in Eq. (3) leads to similar expressions for $\Delta P\left(\mathbf{r}, \mathbf{r}_{s}\right)$ (see Appendix A) when the change comes from introducing/removing a scatterer or from moving one scatterer in the scattering medium. Separating the single scattered wave from the multiple scattering contribution of the subtracted field yields (see Appendix A)

$$
\Delta P\left(\mathbf{r}, \mathbf{r}_{s}\right)=S(\omega) g_{m s} A(\omega) \frac{e^{i k\left(d_{s}+d_{r}\right)}}{\pi k \sqrt{d_{s} d_{r}}}+\Delta N_{m}\left(\mathbf{r}, \mathbf{r}_{s}\right),
$$

where $S(\omega)$ is the source spectrum, $g_{m s}$ is the scattering coefficient of the moving, removed or newly inserted scatterer, $A(\omega)$ is an amplitude factor. Distances $d_{s}$ and $d_{r}$ are calculated from the source to the perturbation location and from the receiver to the perturbation location, respectively, and $\Delta N_{m}$ describes the multiple scattering interactions with the perturbation.

The amplitude factor is such that $A(\omega)=2$ or $A(\omega)=-2$ when inserting or removing a scatterer, respectively, and $A(\omega)=4 \sin (k \delta r(\cos \alpha+\cos \gamma))$ when moving a scatterer. Terms $\alpha$ and $\gamma$ denote the angles between the average location of the moving scatterer and its trajectory as depicted in Fig. 2. The scattering coefficient in Eq. (4) is related to the scattering amplitude ${ }^{19}$ by a factor $\sqrt{2} / \sqrt{\pi k}$. Also, for the sake of clarity, the notation for $g_{m s}\left(\omega, \theta_{m}, \mathbf{r}_{m} \mid \mathbf{r}_{s}\right)$ has been shortened to $g_{m s}$. The scattering amplitude $g_{m s}\left(\omega, \theta_{m}, \mathbf{r}_{m} \mid \mathbf{r}_{s}\right)$ depends on the frequency $\omega$, the scattering direction $\theta_{m}$, and the curvature of the impinging cylindrical wave ${ }^{20}$ on the scatterer at $\mathbf{r}_{m}$ from source at $\mathbf{r}_{s}$. 


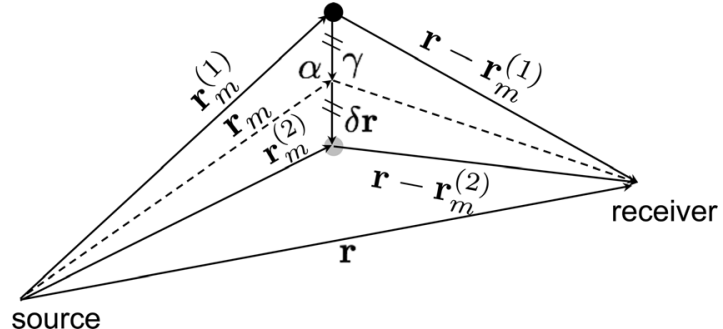

FIG. 2. Scattering configuration between two scatterer positions for one source-receiver pair. The scatterer and the receiver are positioned relatively to the source. The disks represent the two scatterer positions. Subscript (1) refers to the initial scatterer position and subscript (2) to the scatterer position after a small displacement $\delta \mathbf{r}$. The pressure field perturbation is located using the average coordinate $\mathbf{r}_{m}$ between the two scatterer positions.

In the time domain, the subtracted field between the source $s$ and the receiver $r$ is

$$
\begin{aligned}
\Delta p_{s r}(t) & =\frac{1}{2 \pi} \int_{-\infty}^{+\infty} \Delta P\left(\mathbf{r}, \mathbf{r}_{s}\right) e^{i \omega t} d \omega \\
& =\Delta s_{s r}(t)+\Delta n_{s r}(t),
\end{aligned}
$$

where $\Delta s_{s r}(t)$ and $\Delta n_{s r}(t)$ represent the single and multiple scattering interactions with the perturbation, respectively. The expression of the single scattering contribution in the time domain is deduced from Eqs. (4) and (5):

$$
\Delta s_{s r}(t)=e_{s r}(t) * \delta\left(t-\tau_{s r}\right),
$$

where $e_{s r}(t)=T F^{-1}\left[S(\omega) g_{m s} A(\omega)\left(1 / \pi k \sqrt{d_{s} d_{r}}\right)\right], \tau_{s r}$ is the wave travel time from the source to the receiver through the perturbation and is defined as $\tau_{s r}=\left(d_{s}+d_{r}\right) / c$. Neglecting the propagation inside the scatterers, $c$ is the free-space sound speed. The Dirac delta function in Eq. (6) provides a straightforward relation between the wave travel time and the perturbation location. As propagation paths are shorter for single scattering interactions than multiple scattering contributions, the single interaction with the perturbation is associated with the earliest change in the subtracted field (Fig. 3). Consequently, the perturbation location can be recovered from the extraction of this earliest change in the time series and the inversion of the single scattered wave model $\delta\left(t-\tau_{s r}\right)$ in Eq. (6). In the following text, we present

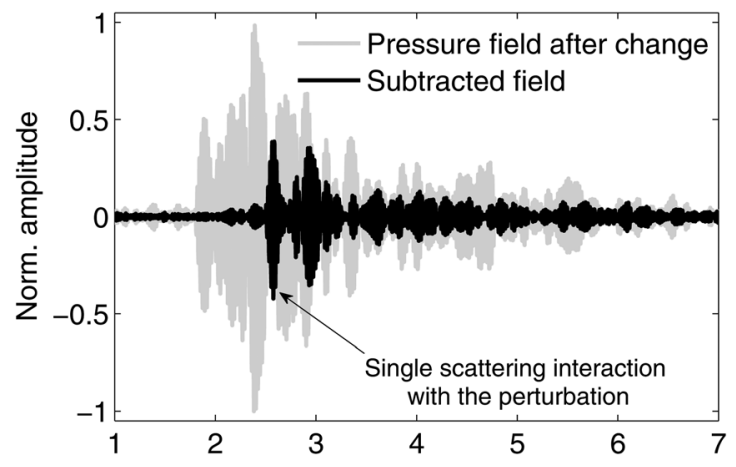

FIG. 3. Point-to-point measurement of the scattered field before the local perturbation and subtracted field before/after the perturbation for the same source-receiver pair. a signal processing method to locate the perturbation that only requires the sound speed of the free space medium and does not require any knowledge of the scattering properties of the multiple scattering medium.

\section{B. Signal processing}

Noting that each candidate cell of the search area at location $\mathbf{r}^{\prime}$ is related to a given arrival time $\tau_{s r}^{\prime}$ of the pressure perturbation $\Delta p_{s r}(t)$, the method calculates the energy variation caused by the perturbation at the steering vector's candidate position, $\mathbf{r}^{\prime}$. Time gating the subtracted field around time $\tau_{s r}^{\prime}$, calculating the energy throughout this time window and summing incoherently over all source-receiver pairs give the following cost function for the position $\mathbf{r}^{\prime}$

$$
\delta \varepsilon_{E}\left(\mathbf{r}^{\prime}\right)=\frac{1}{N_{s}} \frac{1}{N_{r}} \sum_{s=1}^{N_{s}} \sum_{r=1}^{N_{r}} \int_{-\infty}^{+\infty} \Delta p_{s r}(t)^{2} \cdot w\left(t-\tau_{s r}^{\prime}\right) d t
$$

where $N_{s}$ and $N_{r}$ are the number of sources and receivers, respectively, $w\left(t-\tau_{s r}^{\prime}\right)$ is a window of length $\Delta \tau$ centered at $\tau_{s r}^{\prime}$, and $\tau_{s r}^{\prime}$ is the travel time defined as $\tau_{s r}^{\prime}=\left(d_{s}^{\prime}+d_{r}^{\prime}\right) / c$ where $d_{s}^{\prime}$ and $d_{r}^{\prime}$ are the distances from the source and the receiver to the steering coordinate $\mathbf{r}^{\prime}$, respectively. The subtracted field in Eq. (7) is normalized such that $\int_{-\infty}^{+\infty} \Delta p_{s r}^{2}(t) d t=1$ so that all source-receiver pairs have the same weight. Assuming that the scattering medium is surrounded by many sources and receivers, the output of the cost function $\delta \varepsilon_{E}\left(\mathbf{r}^{\prime}\right)$ is at its maximum when the focus spot at $\mathbf{r}^{\prime}$ matches with the perturbation location at $\mathbf{r}_{\boldsymbol{m}}$. As seen in Fig. 4, the windows of the candidate position are located at the earliest wave arrival of the subtracted field if the candidate position fits the actual perturbation location. In the opposite case, some windows from the time gating are positioned on the early part of the signal with zero energy and some energy from later multiple scattering interactions will contribute to the cost function (Fig. 4). Assuming that the length of the single scattered wave is similar to the length of the emitted pulse, the width of the window $\Delta \tau$ is set to $\Delta t$, the size of the transmitted pulse. We note that the subtracted field is related to the variation in the error functional for the Fréchêt derivative given by Norton ${ }^{21}$ and therefore also to

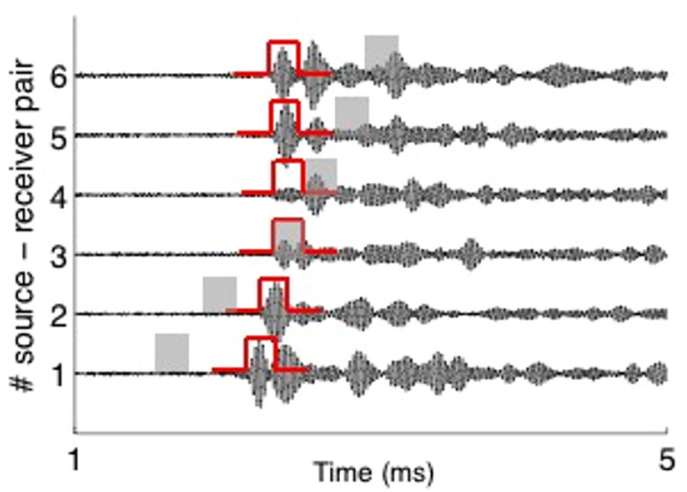

FIG. 4. (Color online) The subtracted field measured over few sourcereceiver pairs. The hats represent the time gating when the process focuses at the actual perturbation location and the rectangle areas locate the windows when the candidate position is not at the actual perturbation location. 
sensitivity kernel analysis. ${ }^{22,23}$ But here the variation in the functional error is given in energy instead of amplitude.

The incoherent summation of the energy throughout the window $w\left(t-\tau_{s r}^{\prime}\right)$ makes this technique insensitive to small random phase shifts from one sensor pair to another in the subtracted field. As mentioned earlier, the scattering coefficient $g_{m s}$ depends on the impinging wave on the scatterer and the scattering direction in the $k a>1$ regime. The phase of $g_{m s}$ varies with source and receiver locations in such a way that the phase of the subtracted field in Eq. (4) is not fully coherent between sensors. This causes any coherent beamforming process to fail at focusing at the target location and therefore suggests the incoherent energy summation over sensor pairs in Eq. (7). As a consequence, the gain of the time gating method is related to the pulse length and to the relative contribution of the single scattering contribution $\Delta s_{s r}$ and the multiple scattering contribution $\Delta n_{s r}$. An estimate of the average signal-to-noise ratio (SNR) between the maximum of the cost function at the actual perturbation location $\mathbf{r}_{\boldsymbol{m}}$ and the rest of the ambiguity surface is given by (see Appendix B)

$$
S N R_{\text {meth }}=10 \log _{10} \frac{1}{\frac{8 \Delta t}{\pi t_{0}}+\left(0.62-\frac{4 \Delta t}{\pi t_{0}}\right) \frac{e_{n}}{e_{s}}},
$$

where $t_{0}$ is the travel time defined as $t_{0}=2 R / c, e_{s}$ is the peak intensity of the single scattered wave, and $e_{n}$ represents the variance of the subtracted field intensity related to multiple scattering contributions. When the pulse width tends to zero, the SNR in Eq. (8) only depends on the ratio between the single and multiple scattering contributions to the subtracted field. The SNR in Eq. (8) is less than or equal to zero if $e_{n}>1.6 e_{s}$. That would happen if the scattering mean free path becomes much smaller than the medium size, ${ }^{24,25}$ which would be the case for the diffuse regime. An estimate of the SNR using Eq. (8) enables one to provide the dynamic range of the expected ambiguity surface discussed in the next section.

\section{EXPERIMENTAL RESULTS}

The experimental setup is depicted in Fig. 1(b). Fifteen electrostatic speakers alternate with 15 condenser microphones. They form a circle around the scattering medium with radius $R=340 \mathrm{~mm}$. The latter is composed of uniformly distributed steel rods with radius $4.75 \mathrm{~mm}$. Each source successively transmits a Gaussian pulse, and the scattered field is measured at each microphone. The field is measured twice over all sensors: once before and once after the local perturbation. Then the difference between the two measured fields is calculated.

First, the method is tested when introducing a new scatterer, a steel rod with radius $a=9.5 \mathrm{~mm}$, into the scattering medium with a density of scatterers $\rho=331 / \mathrm{m}^{2}$. The transmitted pulse is a $\Delta t=0.5 \mathrm{~ms}$ long Gaussian pulse centered at $50 \mathrm{kHz}$. Fifty different perturbation positions within the complex medium are tested. Estimating the transport mean free path in Eq. (9) (Ref. 26) provides $l_{e}=247 \mathrm{~mm}$ at $50 \mathrm{kHz}$, which is about 2.8 times smaller than the diameter of the scattering medium. Note that the mean free path is a function of frequency ${ }^{26}$ although we have omitted the frequency dependence from the notation in Eqs. (9) and (10),

$$
l_{e}=\frac{l_{s}}{1-\overline{\cos (\theta)}},
$$

where $\overline{\cos (\theta)}$ is related to the anisotropy of an individual scatterer ${ }^{26}$ and equals 0.21 for a rigid cylinder with radius $4.75 \mathrm{~mm}$ at $50 \mathrm{kHz}$, and $l_{s}$, the elastic mean free path, is given by

$$
l_{s}=\frac{1}{\rho \sigma_{s}},
$$

where $\sigma_{s}$ is the elastic scattering cross section. An approximate value of $\sigma_{s}=15.5 \mathrm{~mm}$ for a rigid cylinder ${ }^{27}$ is used to represent rods of the scattering medium yielding an elastic mean free path of $195 \mathrm{~mm}$ at $50 \mathrm{kHz}$. The method successfully located the perturbation for 46 locations over the 50 tested locations with an accuracy of a few wavelengths. Over the 46 locations, the actual target position is always embodied in the 0 to $-1 \mathrm{~dB}$ area of the ambiguity surface with an average radius of 4 wavelengths that corresponds to $3 \mathrm{~cm}$. The ambiguity surface for one successful perturbation localization is displayed in Fig. 5.

Next, the method is tested to locate a change caused by moving a scatterer in the scattering medium. The experiment is performed with a higher density of scatterers $\rho=408 / \mathrm{m}^{2}$, and 20 different locations of the perturbation are tested. At each new change location, the moving scatterer is moved 0.5 $\mathrm{cm}$ away from its previous position. The experiment is successively performed at four central frequency values: 30,40 , 50 and $60 \mathrm{kHz}$. The size of the transmitted Gaussian pulse varies from $\Delta t=0.15 \mathrm{~ms}$ at $60 \mathrm{kHz}$ to $\Delta t=0.30 \mathrm{~ms}$ at $30 \mathrm{kHz}$. With the scatterer density of $408 / \mathrm{m}^{2}$, the transport mean free path slowly decreases from $l_{e}=205 \mathrm{~mm}$ at 30 $\mathrm{kHz}$ to $l_{e}=199 \mathrm{~mm}$ at $60 \mathrm{kHz}$. Note that the incident wave has to travel about 3.4 mean free paths from the source to the receiver. The method tracks the moving scatterer and provides a successful estimate of the overall 20 changed locations. Figure 6 shows the ambiguity surface obtained at $30,40,50$, and $60 \mathrm{kHz}$ for one perturbation location. The

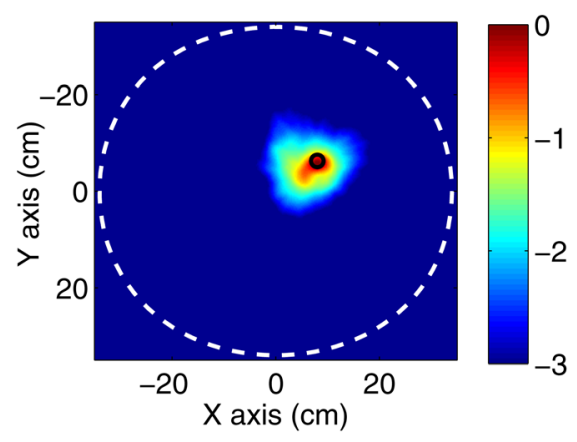

FIG. 5. (Color online) The ambiguity surface in a decibel scale measured at $50 \mathrm{kHz}$ for a new scatterer inserted in the scattering medium delimited by the dashed line circle. The solid line circle represents the actual location of the perturbation and the ambiguity surface is normalized as $10 \log _{10}\left[\left|\delta \varepsilon_{E}\left(\mathbf{r}^{\prime}\right)\right| /\right.$ $\left.\left.\max \mid \delta \varepsilon_{E}\left(\mathbf{r}^{\prime}\right)\right) \mid\right]$ 
a) $30 \mathrm{kHz}$

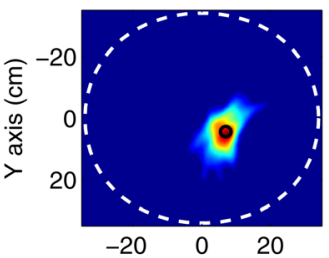

c) $50 \mathrm{kHz}$

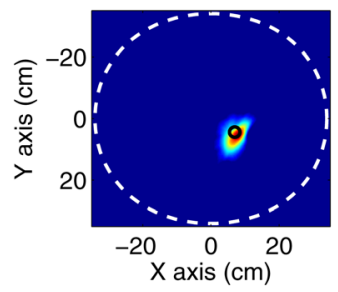

b) $40 \mathrm{kHz}$

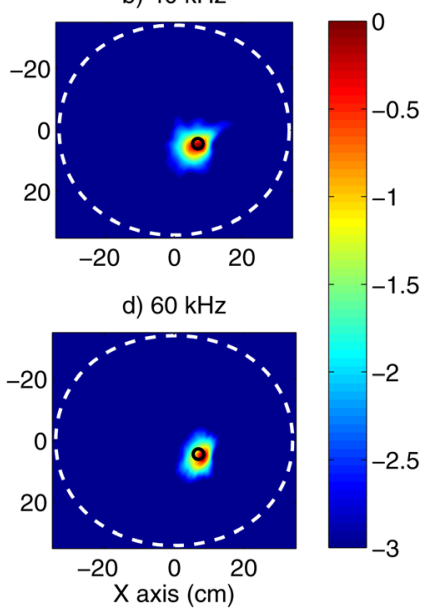

FIG. 6. (Color online) The ambiguity surface in a decibel scale measured at $30,40,50$, and $60 \mathrm{kHz}$ for a perturbation generated by a moving rod in the scattering medium delimited by the dashed line circle. The solid line circle represents the real location of the perturbation and the ambiguity surface is normalized as in Fig. 5.

spatial accuracy of the localization remains almost constant even with a doubled wavelength. The average radius of the 0 to $-1 \mathrm{~dB}$ area (Fig. 6) is $\pm 2.6 \mathrm{~cm}$ over the tested frequencies and the 20 tested locations of the perturbation.

\section{DISCUSSION}

The method presented in this paper is based on the extraction of a single scattered wave from the coherent change. It successfully locates a new target or a moving scatterer assuming that it is the only local perturbation within the scattering medium. Although the scattering medium is about three times larger than the transport mean free path in the tested examples, the method localizes the change because the single scattering interaction with the perturbation is associated with the first change of the subtracted field (Fig. 4). Multiple scattering becomes the only contribution to the subtracted field at later wave arrival times and also participates in the cost function as defined in Eq. (7). This explains the blurry aspect of the ambiguity surface (Figs. 5 and 6). Table I compares SNR predicted by Eq. (8) to SNR from the experiments presented in Sec. III. Estimated values of the SNR in Eq. (8) are consistent with the SNR from experimental data (Table I) and with the dynamic range of the experimental ambiguity surfaces (Figs. 5 and 6). Moreover, the SNR tends to increase with frequency due to the decreasing pulse length. The gain slightly varies from 2.7 to $5 \mathrm{~dB}$ through its dependence on the relative single scattering/multiple scattering contribution $e_{n} / e_{s}$ and the pulse length $\Delta t$.

As the transport mean free path becomes smaller with respect to the medium size, the gain of the method decreases. Experimental tests conducted for a denser medium with a scatterer density $\rho=564 / \mathrm{m}^{2}$ and an approximate transport mean free path $l_{e}=145 \mathrm{~mm}$, show that the method still focuses at the actual perturbation with a lower SNR, although the mean free path is almost five times smaller than the medium size. The method may fail at locating the change if the single interaction with the perturbation is too low rela-
TABLE I. Comparison of SNR from experiments and from the model; $\mathrm{SNR}_{\text {meth }}$ is given by Eq. (8) and $\mathrm{SNR}_{\text {exp }}$ is the average absolute value of cost function over the whole medium excepted at the location of the cost function maximal value.

\begin{tabular}{lcllll}
\hline \hline & New rod & \multicolumn{4}{c}{ Moving rod } \\
\cline { 3 - 6 } & $50 \mathrm{kHz}$ & $30 \mathrm{kHz}$ & $40 \mathrm{kHz}$ & $50 \mathrm{kHz}$ & $60 \mathrm{kHz}$ \\
\hline$e_{n} / e_{s}$ & 0.27 & 0.36 & 0.31 & 0.28 & 0.24 \\
$\mathrm{SNR}_{\text {meth }}(\mathrm{dB})$ & 4.1 & 2.7 & 3.3 & 4.0 & 5.0 \\
$\mathrm{SNR}_{\text {exp }}(\mathrm{dB})$ & 4.8 & 3.3 & 3.9 & 4.8 & 4.8 \\
\hline \hline
\end{tabular}

tive to multiple scattering. This high frequency limitation may happen if the scattering mean free path becomes much smaller than the medium size ${ }^{24,25}$ as is the case in the diffuse regime. Also, the technique works if scattering contributions are strong enough against the background noise to be detected. Given that the scattering cross section increases with frequency, this low frequency limitation applies to wavelengths much larger than the size of the perturbation.

\section{CONCLUSIONS}

The paper presents a method to locate a local change in a strongly scattering medium by analyzing the coherent change caused by the perturbation. The approach uses multiple sensors and does not require any modeling of the multiple scattered field nor any knowledge of the scattering properties of the perturbation. This method finds applications for tracking a moving target in a cluttered environment for nondestructive testing such as crack detection and localization in concrete.

The method's two-step approach consists of (1) extracting the coherent change caused by the perturbation using the difference between the perturbed/unperturbed fields and (2) applying a signal processing method on the subtracted field to locate the local perturbation. The central thrust of the method is based on the inversion of a single scattering model and the fact that the single scattered contribution from the perturbation comes first in the subtracted field. The surrounding source/receiver configuration also improves efficacy of the change localization by uniformly insonifying the entire scattering medium. The method has been successfully tested with a laboratory air setup experiment for a target embedded in a scattering medium the size of which was about two to five transport mean free paths. Experimental results show a successful focus at the change with an accuracy of a few wavelengths $(2 \lambda$ to $4 \lambda$ ) for a large number of different perturbation positions. The efficacy of the approach decreases for very dense or large scattering media that have a transport mean free path much smaller than the size of the scattering medium.

\section{APPENDIX A: DERIVATION OF THE SUBTRACTED FIELD}

A point source at the location $\mathbf{r}_{s}$ transmits a cylindrical wave to the 2-D scattering medium composed of $N$ randomly distributed scatterers. The source, the receiver, and the scatterers are assumed to be in the far-field of each other and $k a \geq 1$, where $a$ is the size of the scatterers and $k$ is the 
wavenumber. The pressure field from the scattering medium at a distant receiver $\mathbf{r}$ from source at $\mathbf{r}_{s}$ is described by the following recursive relations ${ }^{16-18}$

$$
\begin{aligned}
& P\left(\mathbf{r}, \mathbf{r}_{s}\right)=P_{0}\left(\mathbf{r}, \mathbf{r}_{s}\right)+\sum_{j=1}^{N} g_{j i} P_{e x t}\left(\mathbf{r}_{j}, \mathbf{r}_{i}\right) H_{0}\left(k\left|\mathbf{r}-\mathbf{r}_{j}\right|\right), \\
& P_{\text {ext }}\left(\mathbf{r}_{\boldsymbol{n}}, \mathbf{r}_{\boldsymbol{l}}\right)=P_{0}\left(\mathbf{r}_{\boldsymbol{n}}, \mathbf{r}_{s}\right)+\sum_{j=1, j \neq l}^{N} g_{j i} P_{\text {ext }}\left(\mathbf{r}_{\boldsymbol{j}}, \mathbf{r}_{\boldsymbol{i}}\right) H_{0}\left(k\left|\mathbf{r}_{\boldsymbol{n}}-\mathbf{r}_{\boldsymbol{j}}\right|\right),
\end{aligned}
$$

where $P_{0}$ represents the direct field that has not interacted with any scatterer, $P_{\text {ext }}\left(\mathbf{r}_{j}, \mathbf{r}_{i}\right)$ denotes the external field interacting with scatterer at location $\boldsymbol{r}_{j}$ and coming from scatterer located at range $\boldsymbol{r}_{\boldsymbol{i}}, H_{0} \equiv H_{0}^{(1)}$ is the Hankel function, and $g_{j i}$ is the scattering coefficient. For the sake of clarity, $g_{j i}$ is a shorter notation of the scattering coefficient $g_{j i}\left(\omega, \theta_{j}, \mathbf{r}_{j} \mid \mathbf{r}_{i}\right)$, which depends on the frequency $\omega$, the scattering direction $\theta_{j}$ and the curvature of the impinging cylindrical wave ${ }^{20}$ on the scatterer at $\boldsymbol{r}_{\boldsymbol{j}}$ from scatterer or source at $\boldsymbol{r}_{\boldsymbol{i}}$. Also, because the point source transmits a cylindrical wave $P_{0}=H_{0}$.

Next, we consider the pressure field $P_{m}$ containing the scattering events that have interacted with the scatterer $m$. The pressure field $P_{m}$ can be expressed as the contribution of the single scattering interaction with the scatterer $m$ and all multiple scattering interactions between scatterer $m$ and the other scatterers from the scattering medium. Using the same scattering series formalism from Eq. (A1) and the far-field expression of the Hankel function yields

$$
\begin{aligned}
P_{m}\left(\mathbf{r}, \mathbf{r}_{s}\right)= & S(\omega) g_{m s} H_{0}\left(k\left|\mathbf{r}_{\boldsymbol{m}}-\mathbf{r}_{s}\right|\right) \\
& \times H_{0}\left(k\left|\mathbf{r}-\mathbf{r}_{\boldsymbol{m}}\right|\right)+N_{\boldsymbol{m}}\left(\mathbf{r}, \mathbf{r}_{s}\right) \\
= & -g_{m s} \frac{2 S(\omega)}{\pi k} \frac{e^{i k\left(\left|\mathbf{r}_{\boldsymbol{m}}\right|+\left|\mathbf{r}-\mathbf{r}_{\boldsymbol{m}}\right|\right)-i \pi / 2}}{\sqrt{\left|\mathbf{r}_{\boldsymbol{m}}\right|\left|\mathbf{r}-\mathbf{r}_{\boldsymbol{m}}\right|}}+N_{m}\left(\mathbf{r}, \mathbf{r}_{s}\right),
\end{aligned}
$$

where $S(\omega)$ is the source spectrum, $g_{m s}$ is the scattering coefficient of scatterer $m$, and $N_{m}\left(\mathbf{r}, \mathbf{r}_{s}\right)$ represents the multiple scattered wavefield contribution that has interacted with scatterer $m$, and vectors $\mathbf{r}_{\boldsymbol{m}}$ and $\mathbf{r}$ describe the location of the scatterer $m$ and the receiver with respect to the source, respectively.

Two types of perturbation are studied:

(1) the introduction or removal of a scatterer $m$ at location $\mathbf{r}_{\boldsymbol{m}}$ into the scattering medium,

(2) the motion of the scatterer $m$ from location $\mathbf{r}_{m}^{(1)}$ to another location $\mathbf{r}_{\boldsymbol{m}}^{(2)}$ within the scattering medium, all other scatterers remaining fixed.

The index (1) refers to the the unperturbed field before the perturbation has occurred and the index (2) refers to the perturbed field.

\section{A. Introduction/removal of a scatterer in the scattering medium}

When introducing a new scatterer $m$ at $\mathbf{r}_{m}$ into the scattering medium, the unperturbed field does not contain any scattering interactions with the scatterer $m$ so that $P_{m}^{(1)}\left(\mathbf{r}, \mathbf{r}_{s}\right)=0$. The subtracted field is then

$$
\Delta P\left(\mathbf{r}, \mathbf{r}_{s}\right)=-g_{m s} \frac{2 S(\omega)}{\pi k} e^{-i \pi / 2} \frac{e^{i k\left(d_{s}+d_{r}\right)}}{\sqrt{d_{s} d_{r}}}+\Delta N_{m}\left(\mathbf{r}, \mathbf{r}_{s}\right)
$$

where $\Delta N_{m}=N_{m}^{(1)}-N_{m}^{(2)}$, distances $d_{s}$ and $d_{r}$ are denoted $d_{s}=\left|\mathbf{r}_{\boldsymbol{m}}\right|$ and $d_{r}=\left|\mathbf{r}-\mathbf{r}_{\boldsymbol{m}}\right|$.

When removing scatterer $m$ from the scattering medium, the expression of the subtracted field is straightforward and is given by

$$
\Delta P\left(\mathbf{r}, \mathbf{r}_{s}\right)=g_{m s} \frac{2 S(\omega)}{\pi k} e^{-i \pi / 2} \frac{e^{i k\left(d_{s}+d_{r}\right)}}{\sqrt{d_{s} d_{r}}}-\Delta N_{m}\left(\mathbf{r}, \mathbf{r}_{s}\right)
$$

\section{B. Moving target in the scattering medium}

When the perturbation is due to a moving scatterer in the medium, the subtracted field is

$$
\begin{aligned}
\Delta P\left(\mathbf{r}, \mathbf{r}_{s}\right)= & \frac{2 S(\omega)}{\pi k}\left[g_{m s}^{(1)} \frac{e^{i k\left(\left|\mathbf{r}_{m}^{(1)}\right|+\left|\mathbf{r}-\mathbf{r}_{m}^{(1)}\right|\right)}}{\sqrt{\left|\mathbf{r}_{m}^{(1)}\right|\left|\mathbf{r}-\mathbf{r}_{m}^{(1)}\right|}}\right. \\
& \left.-g_{m s}^{(2)} \frac{e^{i k\left(\left|\mathbf{r}_{m}^{(2)}\right|+\left|\mathbf{r}-\mathbf{r}_{m}^{(2)}\right|\right)}}{\sqrt{\left|\mathbf{r}_{\boldsymbol{m}}^{(2)}\right|\left|\mathbf{r}-\mathbf{r}_{m}^{(2)}\right|}}\right] e^{-i \pi / 2}+\Delta N_{\boldsymbol{m}}\left(\mathbf{r}, \mathbf{r}_{s}\right) .
\end{aligned}
$$

Assuming that a small scatterer displacement only produces a small change in its scattering coefficient, we have $g_{m s} \approx g_{m s}^{(1)} \approx g_{m s}^{(2)}$. Following the notation in Fig. 2, the vector $\mathbf{r}_{m}$ is defined as the mean position between two successive scatterer positions and $\delta r=|\delta \mathbf{r}|$ corresponds to the scatterer displacement. Expanding $\left|\mathbf{r}_{\boldsymbol{m}}^{(1)}\right|, \quad\left|\mathbf{r}_{\boldsymbol{m}}^{(2)}\right|, \quad\left|\mathbf{r}-\mathbf{r}_{\boldsymbol{m}}^{(1)}\right|, \quad$ and $\left|\mathbf{r}-\mathbf{r}_{\boldsymbol{m}}^{(2)}\right|$ in terms of the small quantities $\delta r / d_{s}$ and $\delta r / d_{r}$ and neglecting all quadratic terms yields

$$
\begin{aligned}
& \left|\mathbf{r}_{\boldsymbol{m}}^{(1)}\right| \approx d_{s}-\delta r \cos \alpha, \\
& \left|\mathbf{r}_{\boldsymbol{m}}^{(2)}\right| \approx d_{s}+\delta r \cos \alpha, \\
& \left|\mathbf{r}-\mathbf{r}_{\boldsymbol{m}}^{(1)}\right| \approx d_{r}-\delta r \cos \gamma \\
& \left|\mathbf{r}-\mathbf{r}_{\boldsymbol{m}}^{(2)}\right| \approx d_{r}+\delta r \cos \gamma
\end{aligned}
$$

where $\alpha$ and $\gamma$ denote the angles between the average location of the moving scatterer and its trajectory as depicted in Fig. 2. Substituting expressions of $\left|\mathbf{r}_{m}^{(1)}\right|,\left|\mathbf{r}_{m}^{(2)}\right|,\left|\mathbf{r}-\mathbf{r}_{m}^{(1)}\right|$ and $\left|\mathbf{r}-\mathbf{r}_{\boldsymbol{m}}^{(2)}\right|$ into (A5) and neglecting terms $\delta r \cos \alpha$ and $\delta r \cos \gamma$ with respect to $d_{s}$ and $d_{r}$ in the denominator of (A5) yields

$$
\begin{aligned}
\Delta P\left(\mathbf{r}, \mathbf{r}_{s}\right)= & g_{m s} \frac{4 S(\omega)}{\pi k} \sin (k \delta r(\cos \alpha+\cos \gamma)) \frac{e^{i k\left(d_{s}+d_{r}\right)}}{\sqrt{d_{s} d_{r}}} \\
& +\Delta N_{m}\left(\mathbf{r}, \mathbf{r}_{s}\right) .
\end{aligned}
$$

\section{APPENDIX B: GAIN OF THE TIME GATING METHOD}

This appendix examines the signal to noise ratio (SNR) between the maximum of the cost function at perturbation location $\mathbf{r}_{m}$ and the rest of the ambiguity surface: 


$$
S N R_{m e t h}=10 \log _{10} \frac{\delta \varepsilon_{E}\left(\mathbf{r}_{m}\right)}{\overline{\delta \varepsilon_{E}}}
$$

where $\overline{\delta \varepsilon_{E}}$ is the mean value of the cost function over the scattering medium area $\Omega$ (Fig. 1) and is defined by

$$
\overline{\delta \varepsilon_{E}}=\frac{1}{\pi R^{2}} \int_{\Omega} \delta \varepsilon_{E}\left(\mathbf{r}^{\prime}\right) d^{2} \mathbf{r}^{\prime}
$$

Inserting the expression of the cost function defined in Eq. (7) into Eq. (B2) gives

$$
\overline{\delta \varepsilon_{E}}=\frac{1}{\pi R^{2} N_{s} N_{r}} \int_{\Omega} \sum_{s=1}^{N_{s}} \sum_{r=1}^{N_{r}} E\left(\tau_{s r}^{\prime}\right) d^{2} \mathbf{r}^{\prime}
$$

where

$$
E\left(\tau_{s r}^{\prime}\right)=\int_{-\infty}^{+\infty} \Delta p_{s r}(t)^{2} \cdot w\left(t-\tau_{s r}^{\prime}\right) d t
$$

Evaluation of integral (B3) depends on the value of the energy function $E$ in (B4) at any travel time $\tau_{s r}^{\prime}$ associated to the steering vector $\mathbf{r}^{\prime}$ on the area $\Omega$. In the experiment, the sources alternate with receivers and are uniformly distributed around the scattering medium at distance $R$ from its center, and $N=N_{s}=N_{r}$. For simplicity, we also assume that the perturbation occurs at the center of the scattering medium and produces isotropic scattering. With these assumptions, the expression of travel time $\tau_{s r}^{\prime}$ in polar coordinates $\mathbf{r}^{\prime}=(r, \phi)$ is

$$
\begin{aligned}
\tau_{s r}^{\prime}= & \frac{1}{c}\left(\sqrt{R^{2}+r^{2}-2 R r \cos \left(\phi+\phi_{s}\right)}\right. \\
& \left.+\sqrt{R^{2}+r^{2}-2 R r \cos \left(\phi+\phi_{r}\right)}\right),
\end{aligned}
$$

where $\phi_{s}$ and $\phi_{r}$ describe the angular position of source and receiver, respectively. An exact derivation of the SNR value versus scatterer position, sensors number, pulse length, and ratio between $\Delta s_{s r}$ and $\Delta n_{s r}$ cannot be calculated easily. Instead we estimate the SNR for a simplified experimental configuration where the energy of the subtracted field in Eq. (B4) is approximated by two rectangular functions (Fig. 7)

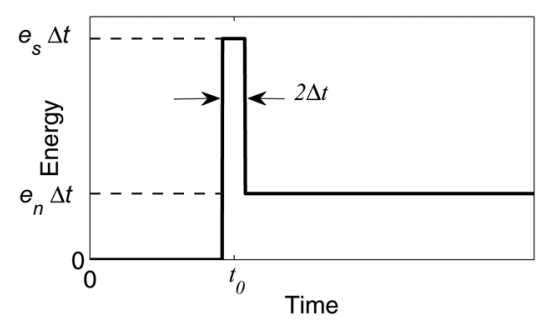

FIG. 7. Simplified version of the subtractedfield energy for a transmitted pulse of length $\Delta t: e_{s}=\Delta s_{s r}^{2}$ is the instantaneous intensity showing the single scattering contribution at $t=t_{0}$ and $e_{n}=\Delta n_{s r}^{2}$ is the instantaneous intensity showing the multiple scattering contribution for $t>t_{0}+\Delta t$ due to the perturbation.
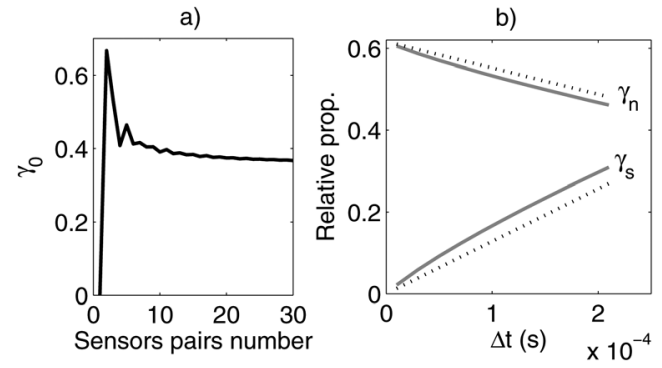

FIG. 8. (a) The average proportion of travel times $\tau_{s r}^{\prime} \in\left[0 ; t_{0}\right]$ over sources and receivers versus the number of sensors pairs; (b) the average proportion $\gamma_{s}$ of travel times $\tau_{s r}^{\prime} \in\left[t_{0}-\Delta t ; t_{0}+\Delta t\right]$ and the average proportion $\gamma_{n}$ of travel times $\left.\left.\tau_{s r}^{\prime} \in\right] t_{0}+\Delta t ; 2 t_{0}\right]$ versus the window size; solid line: numerical evaluation, dashed line: approximate evaluation.

$$
\begin{aligned}
E(t) & =0 \text { for } t<t_{0}-\Delta t \\
& =e_{s} \Delta t \text { for } t_{0}-\Delta t \leq t \leq t_{0}+\Delta t \\
& =e_{n} \Delta t \text { for } t>t_{0}+\Delta t,
\end{aligned}
$$

where $t_{0}$ is the wave travel time from the source to the receiver passing through the perturbation location, $e_{s}=\Delta s_{s r}^{2}$ is the single scattering contribution and $e_{n}=\Delta n_{s r}^{2}$ represents the variance of the subtracted field intensity related to the multiple scattering contributions. Evaluating expression (B3) is equivalent to determining the relative proportions of the travel time $\tau_{s r}^{\prime}$ associated with the energy $E(t)=e_{s} \Delta t$ and $E(t)=e_{n} \Delta t$ over area $\Omega$ :

$$
\overline{\delta \varepsilon_{E}}=\gamma_{s}(\Delta t, R, N) e_{s} \Delta t+\gamma_{n}(\Delta t, R, N) e_{n} \Delta t
$$

where $\gamma_{s}$ is the proportion of travel time $\tau_{s r}^{\prime} \in\left[t_{0}-\Delta t\right.$; $\left.t_{0}+\Delta t\right]$ and $\gamma_{n}$ is the proportion of travel time $\left.\left.\tau_{s r}^{\prime} \in\right] t_{0}+\Delta t ; 2 t_{0}\right]$ over area $\Omega$ and averaged over the $N$ sensors pairs. The upper limit $2 t_{0}$ is the maximum value of $\tau_{s r}^{\prime}$ over the area $\Omega$. To determine $\gamma_{s}$ and $\gamma_{n}$, we first numerically calculate the proportion $\gamma_{0}$ of $\tau_{s r}^{\prime} \in\left[0 ; t_{0}\right]$ over area $\Omega$. The parameter $\gamma_{0}$ depends on the number of sensors and does not depend on the window length $\Delta t$ or the scattering medium size $R$. Further, as shown in Fig. 8(a), the factor $\gamma_{0}$ becomes independent of the number of sensors beyond 10 sourcereceiver pairs and subsequently approaches a constant value of $\gamma_{0} \approx 0.38$. Assuming that there are more than 10 sensors pairs,

(1) $\gamma_{s}$ can be approximated [Fig. 8(b)] using part of the scattering medium covered by the area $2 \Delta t c \times 2 R$ so that $\gamma_{s} \approx 4 \Delta t c / \pi R$, and

(2) $\gamma_{n}$ is well described by $\gamma_{n}=1-\gamma_{0}-\gamma_{s} / 2$ [Fig. 8(b)].

Finally, inserting the expression of $\gamma_{s}$ and $\gamma_{n}$ into the mean cost function of Eq. (B7) and substituting it into the SNR relation in Eq. (B1) yields

$$
\begin{aligned}
S N R_{\text {meth }} & =10 \log _{10} \frac{1}{\gamma_{s}+\gamma_{n} e_{n} / e_{s}} \\
& =10 \log _{10} \frac{1}{\frac{8 \Delta t}{\pi t_{0}}+\left(0.62-\frac{4 \Delta t}{\pi t_{0}}\right) \frac{e_{n}}{e_{s}}} .
\end{aligned}
$$


${ }^{1}$ D. Liu, G. Kang, L. Li, Y. Chen, S. Vasudevan, W. Joines, Q. Huo Liu, J. Krolik, and L. Carin, "Electromagnetic time-reversal imaging of a target in a cluttered environment," IEEE Trans. Antennas Propag. 53, 3058-3066 (2005).

${ }^{2}$ E. Larose, T. Planes, V. Rossetto, and L. Margerin, "Locating a small change in a multiple scattering environment," Appl. Phys. Lett. 96, 204101 (2010)

${ }^{3}$ S. Feng and D. Sornette, "Acoustical nondestructive evaluation of heterogeneous materials in the multiple scattering regime," J. Acoust. Soc. Am. 90, 1742-1748 (1991).

${ }^{4}$ A. Aubry and A. Derode, "Detection and imaging in a random medium: A matrix method to overcome multiple scattering and aberration," J. Appl. Phys. 106, 044903 (2009).

${ }^{5}$ A. Aubry and A. Derode, "Random matrix theory applied to acoustic backscattering and imaging in complex media," Phys. Rev. Lett. 102, 084301 (2009).

${ }^{6}$ A. B. Baggeroer, W. A. Kuperman, and P. N. Mikhalevsky, "An overview of matched field methods in ocean acoustics," IEEE J. Ocean. Eng. 18, 401-424 (1993).

${ }^{7}$ S. E. Dosso and M. J. Wilmut, "Bayesian focalization: Quantifying source localization with environmental uncertainty," J. Acoust. Soc. Am. 121, 2567-2574 (2007).

${ }^{8} \mathrm{C}$. Pacheco and R. Snieder, "Time-lapse travel time change of multiply scattered acoustic waves," J. Acoust. Soc. Am. 118, 1300-1310 (2005).

${ }^{9} \mathrm{M}$. Campillo and A. Paul, "Long-range correlations in the diffuse seismic coda," Science 299, 547-549 (2003).

${ }^{10}$ Y. Ziadé, H. Roussel, M. Lesturgie, W. Tabbara, "A coherent model of forest propagation-application to detection and localization of targets using the dort method," IEEE Trans. Antennas Propag. 56, 1048-3066 (2008).

${ }^{11}$ J. De Rosny and P. Roux, "Multiple scattering in a reflecting cavity: Application to fish counting in a tank," J. Acoust. Soc. Am. 109, 2587-2597 (2001).

${ }^{12}$ D. A. Demer, S. G. Conti, J. De Rosny, and P. Roux, "Absolute measurements of total target strength from reverberation in a cavity," J. Acoust. Soc. Am. 113, 1387-1394 (2002).

${ }^{13}$ R. Snieder, A. Grêt, H. Douma, and J. Scales, "Coda-wave interferometry for estimating nonlinear behavior in seismic velocity," Science 295, 2253-2255 (2002).
${ }^{14}$ O. I. Lobkis and R. L. Weaver, "Coda-wave interferometry in finite solids: recovery of p-to-s conversion rates in an elastodynamic billiard," Phys. Rev. Lett. 90, 254302 (2003).

${ }^{15}$ D. Liu, J. Krolik, and L. Carin, "Electromagnetic target detection in uncertain media: Time-reversal and electromagnetic target detection in uncertain media: Time-reversal and minimum-variance algorithms," IEEE Trans. Geosci. Remote Sens. 45, 934-944 (2007).

${ }^{16} \mathrm{~V}$. Twersky, "Multiple scattering of radiation by an arbitrary configuration of parallel cylinders," J. Acoust. Soc. Am. 24, 42-46 (1952).

${ }^{17}$ L. L. Foldy, "The multiple scattering of waves. I. General theory of isotropic scattering by randomly distributed scatterers," Phys. Rev. 67, 107-119 (1945)

${ }^{18}$ C. M. Linton and P. A. Martin, "Multiple scattering by random configurations of circular cylinders: Second-order corrections for the effective wavenumber," J. Acoust. Soc. Am. 117, 3413-3423 (2005).

${ }^{19}$ A. Ishimaru, Wave Propagation and Scattering in Random Media (IEEE Press and Oxford University Press, New York, 1997), Chap. 2, pp. 9-18.

${ }^{20}$ T. J. Cavicchi and W. D. O'Brien, "Acoustic scattering of an incident cylindrical wave by an infinite circular cylinder," IEEE Trans. Ultrason. Ferroelectr. Freq. Control 35, 78-80 (1988).

${ }^{21} \mathrm{~S}$. J. Norton, "Iterative inverse scattering algorithms: Methods of computing fréchet derivatives," J. Acoust. Soc. Am. 106, 2653-2660 (1999).

${ }^{22}$ J. Sarkar, B. D. Cornuelle, and W. A. Kuperman, "Information and linearity of time-domain, complex-demodulated, amplitude and phase data in shallow water," J. Acoust. Soc. Am. 130, 1242-1252 (2011).

${ }^{23}$ C. Marandet, P. Roux, B. Nicolas, and J. Mars, "Target detection and localization in shallow water: An experimental demonstration of the acoustic barrier problem at the laboratory scale," J. Acoust. Soc. Am. 129, 85-97 (2010).

${ }^{24}$ A. Derode, A. Tourin, and M. Fink, "Random multiple scattering of ultrasound. I. Coherent and ballistic waves," Phys. Rev. E 64, 036605 (2001).

${ }^{25} \mathrm{P}$. Sheng, Introduction to Wave Scattering, Localization and Mesoscopic Phenomena (Springer-Verlag, Berlin, 2006), Chap. 5, pp. 165-170.

${ }^{26}$ A. Tourin, A. Derode, A. Peyre, and M. Fink, "Transport parameters for an ultrasonic pulsed wave propagating in a multiple scattering medium," J. Acoust. Soc. Am. 108, 503-512 (2000).

${ }^{27}$ P. M. Morse and K. U. Ingard, Theoretical Acoustics (Princeton University Press, Princeton, NJ, 1986), Chap. 8, pp. 400-405. 